\title{
The AIDS Autopsy Spleen: A Comparison of the Pre- Anti-Retroviral and Highly Active Anti-Retroviral Therapy Eras
}

\author{
Leslie K. Diaz, M.D., Robert L. Murphy, M.D., John P. Phair, M.D., Daina Variakojis, M.D. \\ Departments of Pathology (LKD and DV) and Medicine (RLM and JPP), Northwestern University Medical \\ School and Northwestern Memorial Hospital, Chicago, Illinois
}

Pathologic findings attributed to human immunodeficiency virus (HIV) infection and acquired immune deficiency syndrome (AIDS) are described in the spleen in studies that encompassed the years preceding highly active anti-retroviral therapy (HAART). Major findings included white-pulp depletion, hemosiderin deposition, spindle cell proliferations, and perivascular hyalinization. Infectious and malignant infiltrates were commonly noted and characterized. The histopathology of AIDS autopsy spleens since the introduction of protease inhibitors has not been studied. Histologic sections from 168 cases of AIDS spleens examined at autopsy over a 19-year period (1982-2000) were evaluated for significant pathologic findings. Multiple morphologic parameters were recorded, including a graded estimation of white-pulp depletion. Significantly less white-pulp depletion was observed in the 39 HAART-era spleens (1995-2000) compared with the 129 spleens from the 1982-1994 patient group $(P=.001)$. The rates of splenic involvement by atypical mycobacteria and cytomegalovirus were similar to those in the past, although the overall clinical rates of these opportunistic infections were found to be decreased. The annual numbers of AIDS autopsies have decreased, reflecting a nationwide trend of lower autopsy rates. Increased survival with HIV infection and AIDS is now apparent. Protease inhibitors, in conjunction with other contemporary therapies, may have contributed to the preservation of white pulp observed in the HAART patient group.

Copyright (C) 2002 by The United States and Canadian Academy of Pathology, Inc.

VOL. 15, NO. 4, P. 406, 2002 Printed in the U.S.A.

Date of acceptance: November 7, 2001.

This study was partially funded by National Institutes of Health Grant AI35039.

Address reprint requests to: Daina Variakojis, M.D., Northwestern University Medical School, Northwestern Memorial Hospital, Department of Pathology, Feinberg 7-325, 251 E. Huron St., Chicago, IL 60611; e-mail: d-variakojis@northwestern.edu.
KEY WORDS: Acquired immunodeficiency syndrome, Autopsy, Highly active anti-retroviral therapy, Human immunodeficiency virus, Lymphoma, Pneumocystis carinii infections, Spleen.

Mod Pathol 2002;15(4):406-412

Pathologic findings in the spleens of acquired immune deficiency syndrome (AIDS) patients have been characterized in a small number of studies and case reports that were performed before the widespread use of highly active anti-retroviral therapy (HAART; 1-6). HAART regimens consist of various combinations of nucleoside reverse transcriptase inhibitors, nonnucleoside reverse transcriptase inhibitors, and protease inhibitors. Standard HAART regimens use three to four agents and usually include at least a single protease inhibitor (7). Protease inhibitors have prolonged overall survival in AIDS patients and may act to decrease lymphocyte destruction. Introduced in 1995, protease inhibitors were restricted to clinical trials until late 1996, when they became widely available. Recently, it has been demonstrated that protease inhibitors stimulate hematopoiesis and decrease apoptosis of CD34+ cells (8). These progenitor cell-preserving properties of protease inhibitors appear to be independent of their direct antiviral activity. Increased numbers of CD4 + T cells contribute to a partial restoration of the immune system in treated patients, a phenomenon observed since the institution of HAART (9).

In the spleen, microscopically observable whitepulp depletion has been attributed directly to HIV infection and is thought to represent a severe reduction in the number of $\mathrm{T}$ cells and an impairment in macrophage function $(6,10,13)$. Specific histologic findings attributed to HIV in the spleen include amyloid-like protein deposition, perivascular hyalinization, infarcts, necrosis, hemosiderin pigment deposits, plasma cell infiltrates, neutrophilic microabscesses, spindle cell infiltrates, extramedullary hematopoiesis, and granulomatous reaction (2, 
$6,10-13)$. Some of these changes have been shown to be associated with specific infections (2). Our aim in this study was to compare AIDS splenic pathology in a more recent group of patients with splenic pathology in AIDS before the use of multiagent therapy (i.e., the pre-HAART era, 1982-1994).

\section{MATERIALS AND METHODS}

From the files of the autopsy service of Northwestern Memorial Hospital, Chicago, Illinois, 168 AIDS autopsies with complete reports and histologic sections were identified over a 19-year period (1982-2000). One hundred twenty-nine cases constituted the pre-HAART cohort (1982-1994), and 39 cases, from 1995 to the end of 2000, comprised the HAART cohort. Detailed hematoxylin and eosin slide review was performed by two pathologists (LKD and DV) using previously published studies as guidelines. Each patient carried an antemortem diagnosis of AIDS as defined by CD4 count or by an AIDS-defining illness. Treatment histories, CD4 counts, viral loads, and physical examination data were obtained when available. Histochemical as well as immunohistochemical stains were examined when applicable. All morphological parameters were systematically recorded.

On the basis of histologic descriptions in the literature $(2,14)$ and of our experience with surgical and non-AIDS autopsy spleens used as controls, a semiquantitative grading system measured the amount of white-pulp depletion. The grading was performed by examining a single entire histologic section at low power ( $4 \times$ objective) and calculating the total estimated area of white pulp over the total estimated area of red pulp. We considered a normal white-pulp percentage (in a healthy individual) to be $20 \%$. The grading was as follows: Grade $0,<1 \%$ total area containing white pulp; Grade 1, $1-5 \%$ white pulp; Grade 2, 6-10\% white pulp; Grade 3, $11-20 \%$ white pulp; Grade 4, $21-30 \%$ white pulp. In cases with severe sinusoidal congestion ( $n=77 ; 57$ pre-HAART, 20 HAART), the area of red pulp was decreased by $50 \%$ for the calculation. Accordingly, the grades were assigned the following descriptive categories: Grade 0, total white-pulp absence; Grade 1, severe white-pulp depletion; Grade 2, moderate white-pulp depletion; Grade 3, mild depletion to normal white-pulp amounts; and Grade 4, abundant white pulp. Statistical analysis was performed using Analyze-it statistical software (Analyze-It Software, Ltd., Leeds, UK) and a twotailed unpaired Student's $t$ test (www.statpages.net). Additional epidemiologic data gathered from these two cohorts were also recorded.

\section{ANNUAL NUMBERS OF AIDS AUTOPSIES}

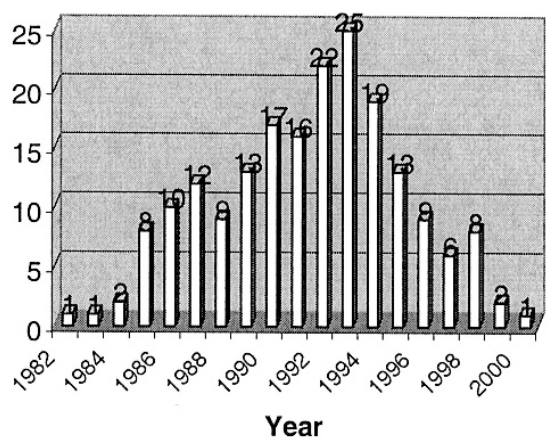

FIGURE 1. Annual numbers of acquired immune deficiency syndrome autopsies performed at Northwestern Memorial Hospital (Chicago, IL) from 1982-2000.

\section{RESULTS}

The total numbers of AIDS autopsies at our institution (Northwestern Memorial Hospital) over the past 19 years are displayed in Figure 1. The rates of AIDS autopsies have markedly declined since their peak in 1993. Figure 2 charts the ages and sexes of the patients studied. All but 12 of the patients were male. The first female was autopsied in 1987, and 10 of the additional female autopsies occurred after 1992. The mean age of autopsied patients has increased since 1997, although the numbers of these patients are relatively small compared with the pre1995 group. The single patient autopsied in the year 2000 died at 60 years of age.

Figure 3 displays the rates of morphologic findings and compares the pre-HAART and HAART patients. The most frequently observed pathologic findings were granulomatous reaction, plasma cell infiltrates, amyloid-like deposits, perivascular hyalinization, and white-pulp depletion. Additional observations included hemosiderin pigment seen within macrophages, spindle-cell infiltrates, necro-

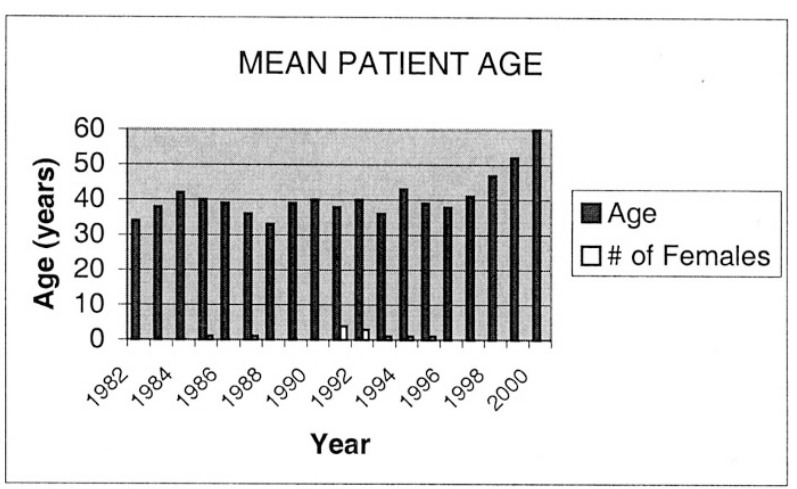

FIGURE 2. Mean patient age per year of autopsied patients (mean age in years) and the number of autopsies performed on females each year (number of cases). 
sis, acute inflammation, and extramedullary hematopoiesis. These findings were relatively common in both patient groups with similar rates of inflammatory infiltrates. Perivascular hyalinization was more frequently observed in the pre-HAART spleens. The HAART spleens displayed a slightly higher incidence of amyloid-like proteinaceous material. The differences in the morphologic characteristics between the two patient groups were not statistically significant. These various pathologic findings have been addressed in previous studies, where their possible meanings are discussed $(2,10-13)$.

The rates of splenic involvement and clinical rates of the major opportunistic infections and malignancies associated with AIDS are shown in Figure 3. Splenomegaly is thought to be a common clinical finding in AIDS patients with infectious or malignant infiltrates. Figure 4 charts the mean weight of the spleens at the time of autopsy by year. Splenic weights remained relatively constant during the 19 years studied. Forty percent of the spleens in this study weighed $>300 \mathrm{~g}$, but clinical enlargement was documented in only 10 cases (2\%). Table 1 lists the clinical characteristics and secondary diseases of these 10 patients. Table 2 lists the findings in 19 patients whose splenic weights

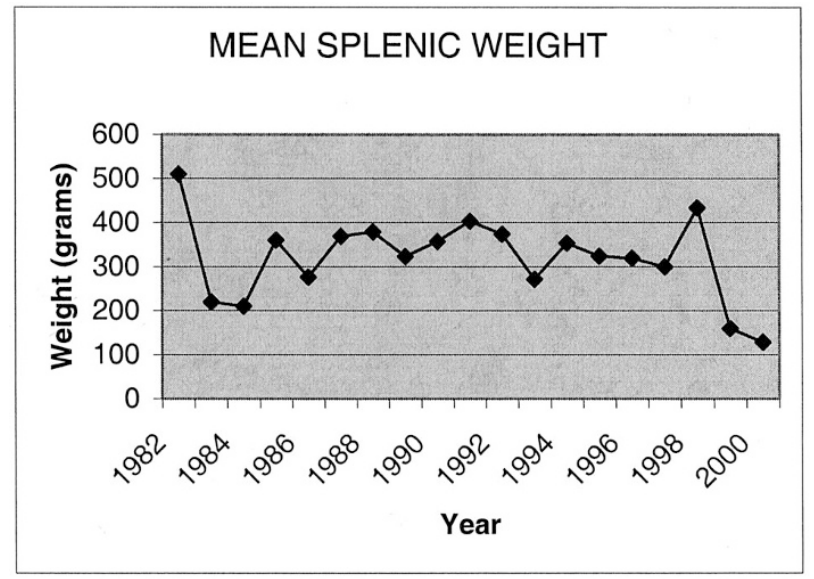

FIGURE 4. Mean splenic weight per year in grams for acquired immune deficiency syndrome autopsies.

were $>600 \mathrm{~g}$. Massive splenomegaly of $>1000 \mathrm{~g}$ was observed in three patients; two of these spleens were enlarged by severe sinusoidal congestion, and the third was extensively infiltrated by large cell lymphoma.

The rates of clinical infection by each agent recorded-cytomegalovirus (CMV), mycobacterium avium intracellulare (MAI), and Pneumocystis cari-

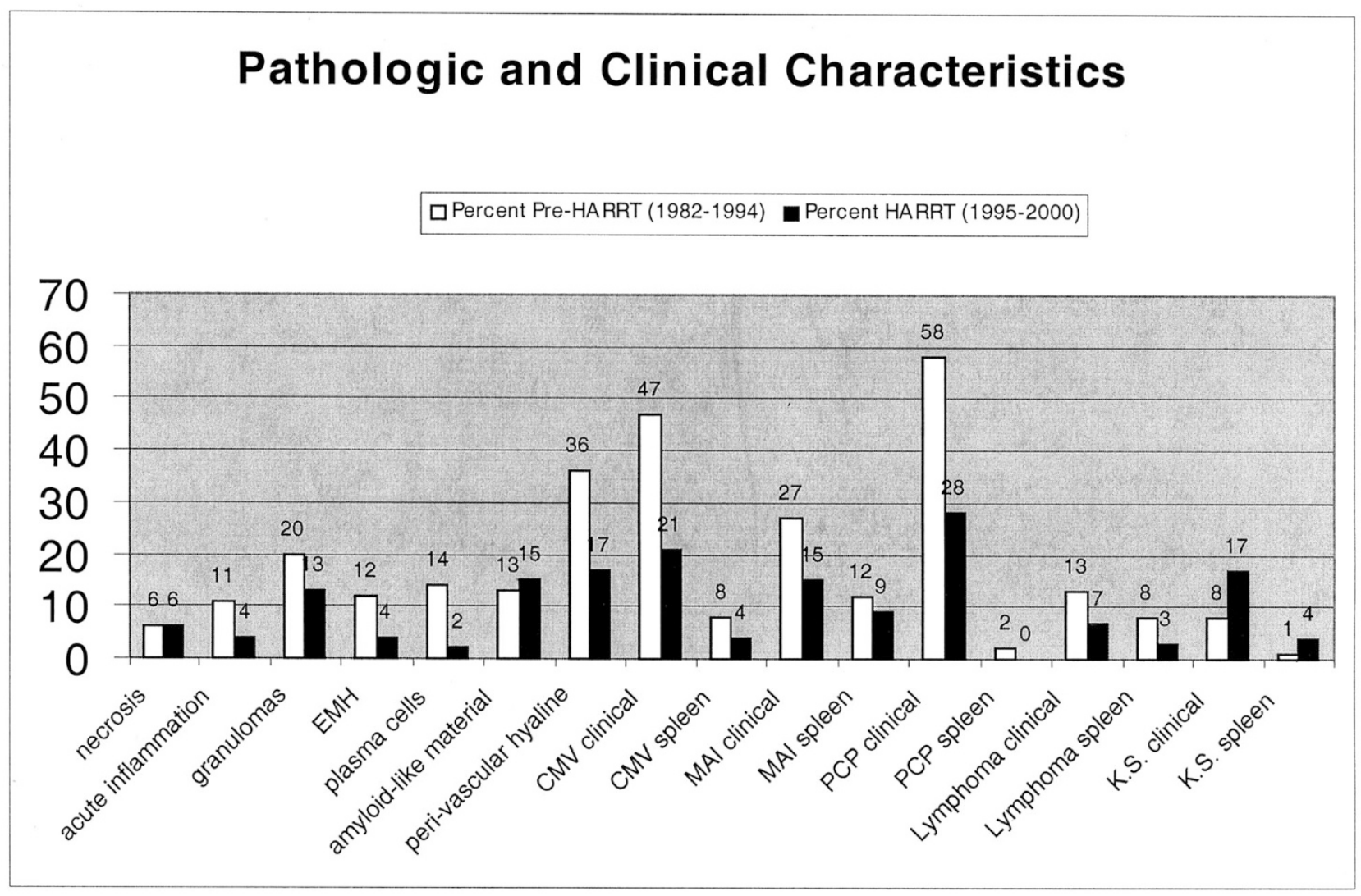

FIGURE 3. The pathologic and clinical characteristics of the studied acquired immune deficiency syndrome autopsy cohorts: pre-highly active anti-retroviral therapy (HAART; 1982-1994) and HAART (1995-2000). Numbers represent percentages of patients within a respective cohort. EMH, extramedullary hematopoiesis; K.S., Kaposi's sarcoma. 
TABLE 1. Characteristics of Patients with Clinical Splenomegaly

\begin{tabular}{|c|c|c|c|c|c|c|c|}
\hline Pt. No. & Year & Age (y) & Cohort & Weight (g) & Clinical Diagnosis & Spleen Involvement & Treatment \\
\hline 1 & 1982 & 34 & pre-HAART & 510 & CMV & no & N/A \\
\hline 2 & 1989 & 40 & pre-HAART & 650 & MAI, CMV, PCP & CMV, PCP & N/A \\
\hline 3 & 1990 & 52 & pre-HAART & 280 & PCP & no & AZT \\
\hline 4 & 1992 & 41 & pre-HAART & 370 & CMV, PCP & no & AZT \\
\hline 5 & 1992 & 46 & pre-HAART & 1290 & large cell lymphoma & yes & N/A \\
\hline 6 & 1993 & 48 & pre-HAART & 140 & CMV, PCP & PCP & N/A \\
\hline 7 & 1993 & 34 & pre-HAART & 750 & MAI & No & AZT \\
\hline 8 & 1994 & 42 & pre-HAART & 700 & CMV, MAI, KS & MAI, KS & MA \\
\hline 9 & 1996 & 35 & HAART & 720 & large cell lymphoma & Yes & MA \\
\hline 10 & 1997 & 40 & HAART & 610 & CMV, MAI & CMV, MAI & no \\
\hline
\end{tabular}

CMV, cytomegalovirus; MAI, mycobacterium avium intracellulare; PCP, pneumocystis carinii pneumonia; Ly, lymphoma; MA, multiagent therapy; KS, Kaposi's sarcoma; HAART, highly active antiretroviral therapy; N/A, not available; AZT, azidothymidine

TABLE 2. Spleens > $600 \mathrm{~g}$. Pathologic and Clinical Findings

\begin{tabular}{lccc}
\hline Splenic Weights $(\mathrm{g})$ & No. of patients & Pre-HAART/HAART & Causes of Splenomegaly $(n)$ \\
\hline$>600-700$ & 7 & $5 / 2$ & $\begin{array}{l}\text { Blasto (1), MAI (2), large cell lymphoma } \\
(1), \text { congestion (3) }\end{array}$ \\
$>700-800$ & 7 & $1 / 6$ & MAI (2), KS (1), large cell lymphoma (1), \\
& & & congestion (3) \\
$>800-1000$ & 2 & $2 / 0$ & Crypto (1), congestion (1) \\
$>1000 \mathrm{gm}$ & 3 & $3 / 0$ & Large cell lymphoma (1), congestion (2) \\
\hline
\end{tabular}

Blasto, blastomycosis; MAI, mycobacterium avium intracellulare; KS, Kaposi's sarcoma; Crypto, cryptococcus; congestion, sinusoidal congestion.

nii pneumonia (PCP)—-decreased in the HAART group, with the largest decrease seen in the incidence of clinical PCP infection (28\% HAART, 58\% pre-HAART). The decreased rate of clinical PCP infection in the HAART patient group was the only statistically significant difference observed for infectious processes $(P=.006)$. The rates of MAI $(15 \%$ HAART, $27 \%$ pre-HAART) and CMV (21\% HAART, $47 \%$ pre-HAART) clinical infections were also found to be decreased in the HAART group; however, the rates of splenic involvement by these agents were similar in affected patients. Disseminated extrapulmonary PCP infection, including splenic involvement, was observed in two pre-HAART patients. A decrease in the rate of AIDS-associated lymphoma was observed in the HAART group (7\%) compared with the pre-HAART group (13\%). The rate of Kaposi's sarcoma was higher clinically in the HAART group ( $17 \%$, versus $8 \%$ pre-HAART).

The mean white-pulp grade was increased in patients from the HAART era (1995-2000) compared with those from the pre-HAART-era (1982-1994), indicating less depletion of white pulp in the HAART-era patients. Mean white-pulp grades were significantly different in these two groups: 2.4 (HAART), versus 1.5 (pre-HAART; $P=.001$ ). The mean CD4 counts in the pre-HAART cohort were 60 cells $/ \mathrm{mm}^{3}$, compared with $88 \mathrm{CD} 4$ cells $/ \mathrm{mm}^{3}$ in the HAART cohort. On a case by case basis, there appeared to be some correlation between the number of CD4 $+\mathrm{T}$ cells present nearest to death and the white-pulp grade; however, the white pulp was significantly preserved in the HAART cohort as a whole. The viral load data that was available did not show a correlation with white-pulp grade.
The white-pulp grades in the years before azidothymidine (AZT) therapy (1982-1989) were not significantly different from the 1990-1994 subset of pre-HAART patients. Forty-four percent of the HAART-era patients (1995-2000) were confirmed to have received multiagent therapy including a protease inhibitor, and although unavoidable, this most likely represents an underestimation of the actual number of patients receiving multiagent therapy. Significant differences in the rates of histologic findings, opportunistic infections, and malignancies were not observed in these patients. The majority of the patients without confirmed protease inhibitor use did receive multiple nucleoside analogs with or without nonnucleoside reverse transcriptase inhibitors. The white-pulp grades were not significantly different in these patients when compared with HAART-era patients without documented multiagent therapy use $(P=.10)$.

\section{DISCUSSION}

Splenic white-pulp depletion has been described and morphologically characterized in previous studies that took place before the widespread use of protease inhibitors as part of HAART or "combination therapy" $(1,2,6,10,13)$. It is thought that white-pulp depletion represents a significant reduction in the number of T cells as a result of direct viral effects; however, B cells may also be affected secondarily. White-pulp depletion (Fig. 5) in the pre-HAART group of spleens generally consisted of a marked reduction in the quantity of lymphocytes with total, to near total, loss of normal follicles. 


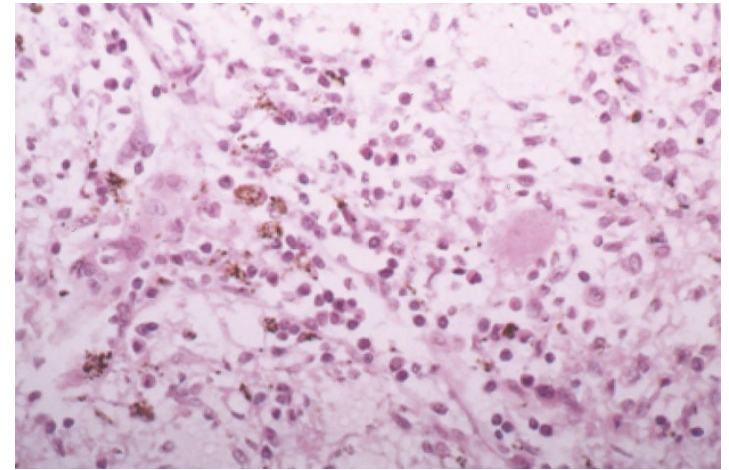

FIGURE 5. Medium-power view of marked white-pulp depletion in a pre-highly active anti-retroviral therapy-era spleen showing loss of follicles and hemosiderin present in macrophages.

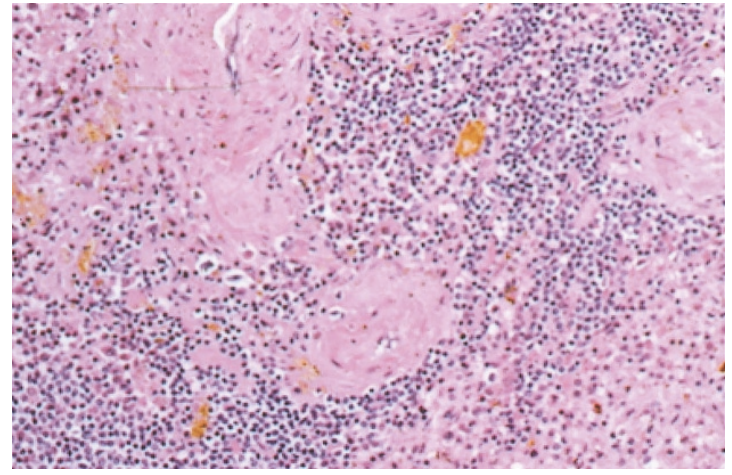

FIGURE 7. Medium-power view of a spleen with moderate whitepulp depletion with disruption of the follicular architecture and presence of hemosiderin pigment.

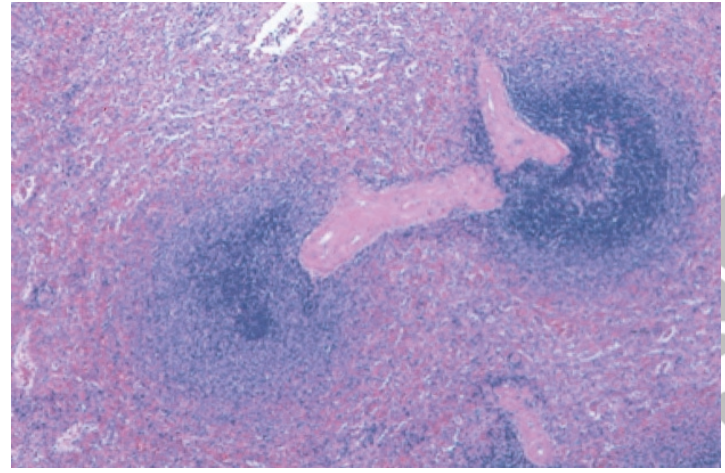

FIGURE 6. Low-power view of normal spleen used for comparison showing intact, abundant white pulp.

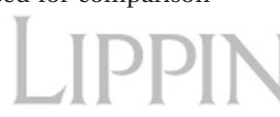

Non-AIDS spleens used for comparison (Fig. 6) possessed intact follicular white-pulp architecture with frequent germinal center formation. Spindleshaped cells were often seen in place of follicles in the AIDS spleens. These cells are thought to be remnants of the dendritic reticulum cell network and macrophages $(10,13)$.

Higher levels of white-pulp preservation were observed in the HAART-era patients, with the overall spectrum of morphologic findings ranging from severe depletion to nearly normal-appearing spleens. The most common morphologic picture was a spleen with a somewhat disrupted follicular architecture and moderately to mildly reduced numbers of lymphocytes (Figs. 7-8). Germinal centers were noted in a minority of cases. We hypothesize that protease inhibitors may be responsible for this observed preservation of splenic white pulp in AIDS autopsy patients since 1995 and that this finding should reflect better preservation of immune function in other hematopoietic locations, including the lymph nodes and bone marrow, organs not always sampled at autopsy.

In detailed work by Klatt and Meyer (2) in 1987, the morphologic findings observed in AIDS spleens were characterized early in the course of the epidemic. Splenic changes were categorized according

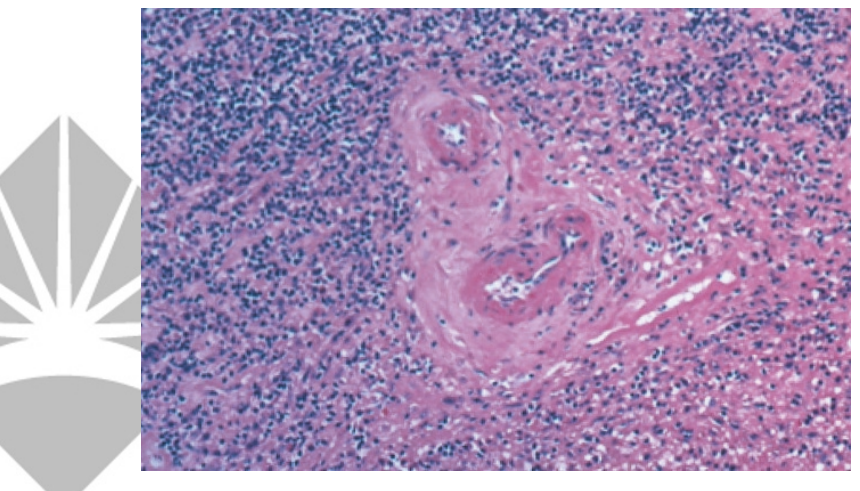

FIGURE 8. Medium-power view showing mild white-pulp depletion of a highly active anti-retroviral therapy-era spleen.

to white-pulp and red-pulp patterns. It was shown that lymphoid depletion and histiocytic (spindle cell) proliferations were more frequently associated with opportunistic infections. We did not analyze our cases in this fashion; however, we observed a similar spectrum of morphologic findings in both of our patient cohorts. Furthermore, we confirmed more curious findings in the literature, such as perivascular hyaline fibrosis (possibly an exaggeration of normal splenic histology, Fig. 9), splenic "para-amyloid" material, and spindle cell prolifer-

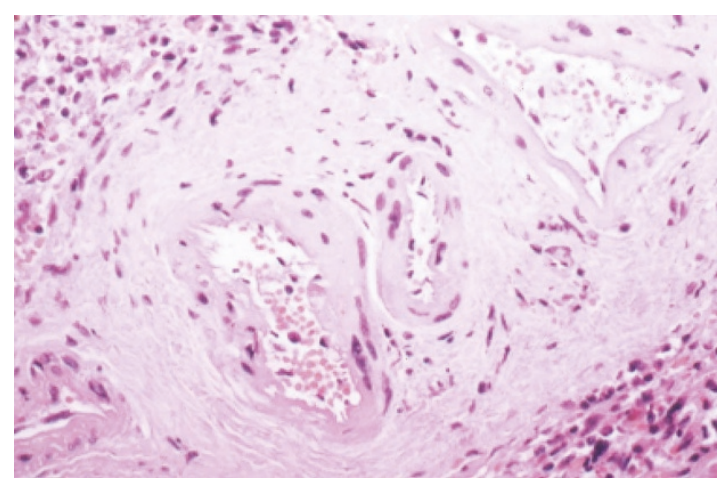

FIGURE 9. Perivascular hyalinization, a relatively frequent finding in both patient groups, represents an exaggeration of the normal splenic architecture. 
ations $(2,5,10,12,15)$. Inflammatory infiltrates, also previously described, were observed with some frequency in both patient groups in this study, including granulomas, plasma cells, extramedullary hematopoiesis, and neutrophilic microabscesses. Only granulomatous reactions were frequently associated with specific infections of the spleens, usually MAI, and rarely M. tuberculosis or fungi.

Interesting demographic data was generated as a result of this study. The rates of AIDS autopsies were found to have markedly decreased since a peak, at our institution, of 25 annual cases in 1993. We believe that this reflects a nationwide trend in the reduction of all autopsies, and especially of human immunodeficiency virus (HIV)-positive patients $(15,16)$. AIDS patients are now more likely than before to die at home or in hospice care as opposed to in an inpatient setting. Some institutions are currently deferring postmortem examinations of HIV-positive individuals. Moreover, it is now clear that patients are dying later with the disease (9). The mean age of autopsied patients steadily increased since 1996. Patients are living longer with AIDS, and studying these patients to determine their clinical courses and outcomes will yield important data in the future.

A pressing question that was addressed but not answered by this study is an accurate determination of the current incidence of AIDS-related lymphomas (Fig. 10). A small decrease in the clinical rate of lymphomas in the HAART-era patients was found in this study (7\%, compared with $13 \%$ preHAART), with splenic involvement by lymphoma seen in approximately one-half of each patient group. The findings currently reported in the literature are nonconclusive, and the use of HAART has not been shown to reduce the incidence or increase the survival of AIDS-related lymphomas (17). One possible outcome of increased overall survival with AIDS is a longer timeframe in which the development of a lymphoproliferative process could occur $(18,19)$. A recent AIDS autopsy study of 390 cases

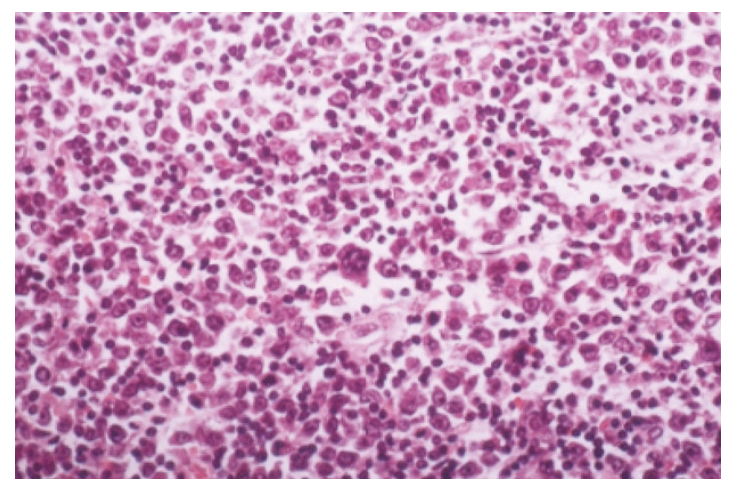

FIGURE 10. Large cell lymphoma involving a pre-highly active antiretroviral therapy spleen. from 1982 to 1998 showed an upward trend in nonHodgkin's lymphoma over time (20). Further investigation will be needed this decade on large groups of HIV-positive patients receiving HAART to accurately establish the prevalence of AIDS-related lymphomas.

In summary, the AIDS autopsy is becoming a rarer event in the hospital setting, especially since the late 1990s and since the widespread institution of HAART. The rates of opportunistic infections are decreasing, and PCP continues to be the most common opportunistic infection. Bacterial pneumonia has recently been shown to be the most frequent pulmonary complication in AIDS (21). HIV-infected patients are living longer, apparently because of increased overall immune function. This "restoration" of immunity, we found, is reflected in the spleens of treated patients by an increased preservation of white pulp. More investigations, both clinical and pathologic, will be required to assess the outcomes of treated patients (22). AIDS patients may now be living for a decade or more with the disease, and AIDS-related lymphomas may rival opportunistic infections as a leading cause of death in the future.

\section{REFERENCES}

1. Falk S, Muller H, Stutte HJ. The spleen in immunodeficiency syndrome (AIS). Pathol Res Pract 1988;183:425-33.

2. Klatt EC, Meyer PR. Pathology of the spleen in acquired immunodeficiency syndrome. Arch Pathol Lab Med 1987; 111:1050-3.

3. Klatt EC, Nichols L, Noguchi TT. Evolving trends revealed by autopsies of patients with the acquired immunodeficiency syndrome. 565 autopsies in adults with the acquired immunodeficiency syndrome. Arch Pathol Lab Med 1994;118:884-90.

4. Mirchandani HG, Mirchandani IH, Pak MS. Spontaneous rupture of the spleen due to acquired immunodeficiency syndrome in an intravenous drug abuser. Arch Pathol Lab Med 1985;109:1114-6.

5. Seshi B. Two cases of AIDS with florid Mycobacterium avium-intracellulare infection in the T-cell areas of the spleen. Hum Pathol 1985;16:964-5.

6. Quijano G, Siminovich M, Durt R. Histopathologic findings in the lymphoid and reticuloendothelial system in pediatric HIV infection: a postmortem study. Pediatr Pathol Lab Med 1997;17:845-56.

7. Yamashita TE, Phair JP, Munoz A, et al. Immunologic and virologic response to highly active antiretroviral therapy in multicenter AIDS cohort study. AIDS 2001;15:735-46.

8. Soland EM, Maciejewski J, Kumar P, et al. Protease inhibitors stimulate hematopoiesis and decrease apoptosis and ICE expression in CD34+ cells. Blood 2000;96:2735-9.

9. Lederman MM, Valdez H. Immune restoration with antiretroviral therapies. JAMA 2000;284:223-8.

10. Falk S, Stutte HJ. The spleen in HIV infection-morphological evidence of HIV-associated macrophage dysfunction. Res Virol 1990;141:161-9.

11. Thomas PA, Anderson VM, Greco MA. Pathology of the spleen. In: Moran C, Mullick FG, editors. Armed Forces Institute of Pathology Fascicle FS16. Systemic pathology of HIV infection and AIDS in children. Washington, D.C.: Armed Forces Institute of Pathology, 1997. pp. 237-49. 
12. Markowitz GS, Factor SM, Borczuk AC. Splenic para-amyloid material: a possible vasculopathy of the acquired immunodeficiency syndrome. Hum Pathol 1998;29:371-6.

13. Falk S, Stutte HJ. The spleen in HIV infection-morphological evidence of HIV-associated macrophage dysfunction. Res Virol 1990;141(2):161-9.

14. van Krieken J, te Velde J. Normal histology of the spleen. Am J Surg Pathol 1998;12:777-85.

15. Lundberg GD. Severed trust: why American medicine hasn't been fixed. New York: Basic Books; 2000.

16. Mitchell EK, Prior JT. Where have all the autopsies gone? A proposal for a centralized autopsy service. J Community Health 1995;20:441-6.

17. Matthews GV, Bower M, Mandalia S, et al. Changes in acquired immunodeficiency syndrome-related lymphoma since the introduction of highly active antiviral therapy. Blood 2000;96:2730-4.
18. Swinnen LJ. Transplantation-related lymphoproliferative disorder: a model for human immunodeficiency virusrelated lymphomas. Semin Oncol 2000;27:402-8.

19. Tao J, Wasik M. Epstein-Barr virus associated polymorphic lymphoproliferative disorders occurring in nontransplant settings. Lab Invest 2001;81:429-37.

20. Masliah E, DeTeresa RM, Mallory ME, Hansen LA. Changes in pathological findings at autopsy in AIDS cases for the last 15 years. AIDS 2000;14:69-74.

21. Bekele A, Green W, Chiao J, Frederick W. Pulmonary complications of HIV infection: autopsy findings. Chest 1998;113: 1225-9.

22. Valdez H, Chowdhry TK, Asaad R, et al. Changing spectrum of mortality due to human immunodeficiency virus: analysis of 260 deaths during 1995-1999. Clin Infect Dis 2001;32: 1487-93.

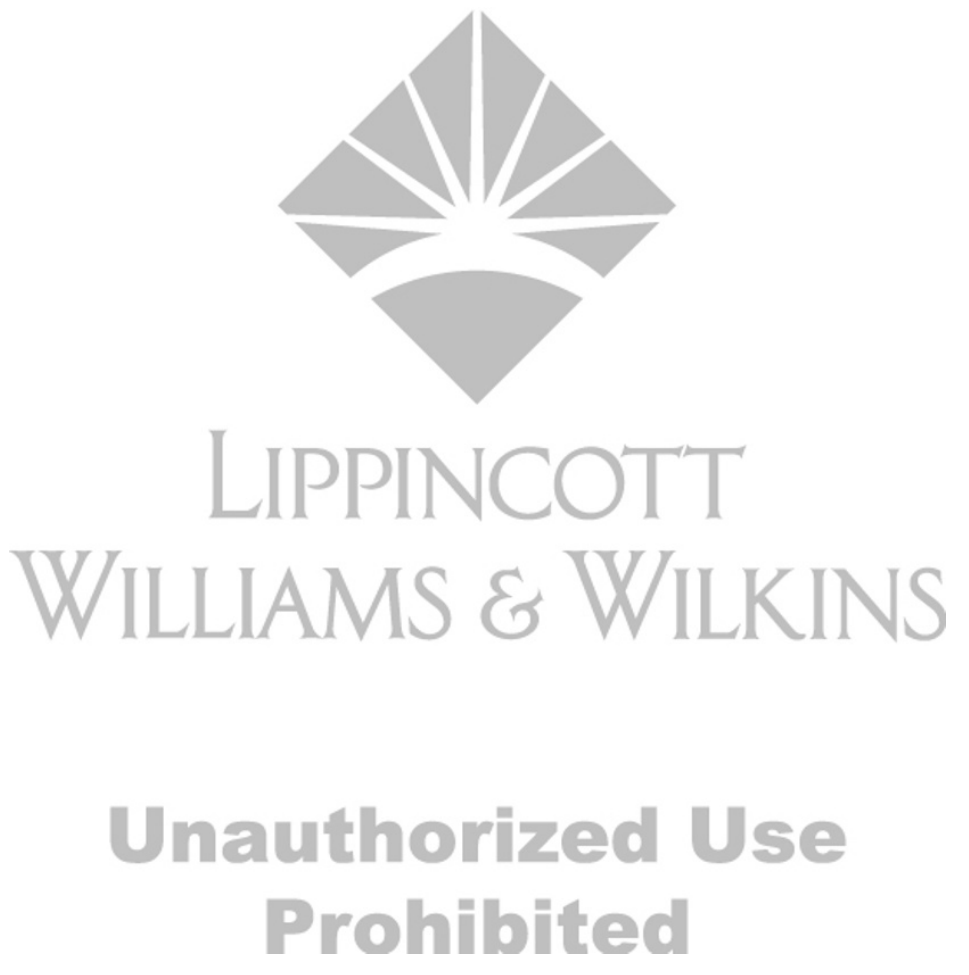

